Communications in Physics, Vol. 24, No. 3S2 (2014), pp. 146-150

DOI:10.15625/0868-3166/24/3S2/5012

\title{
THERMODYNAMIC EQUIVALENT BETWEEN NON- INTERACTING BOSE AND FERMI GAS IN METALLIC CARBON NANOTUBES
}

\author{
PHAM THI KIM HANG, CHU THUY ANH, PHAM VAN DIEN, TRAN THI THANH VAN, \\ NGUYEN TRI LAN AND NGUYEN AI VIET \\ Institute of Physics, Vietnam Academy of Science and Technology \\ E-mail: ctanh@iop.vast.ac.vn
}

Received 20 June 2014

Accepted for publication 20 August 2014

\begin{abstract}
The equivalent between Bose and Fermi ideal gases is usually taken in high temperature limit only. Recently, there has been considerable interest in surprising thermodynamic "equivalences" between certain ideal Bose and spineless Fermi gas systems in lower temperature. In this work, we follow that idea to investigate the quasi onedimensional system of metallic carbon nanotubes. Due to the linear dispersion law, the non-interacting Bose and Fermi gases in metallic carbon nanotubes are equivalent. This equivalence could be applied to the gas systems of exciton photon (Bose particles) and electron hole (Fermi particles) in metallic carbon nanotubes.
\end{abstract}

Keywords: carbon nanotube, statistic physics, nano material, distribution function.

\section{INTRODUCTION}

We all know that they are quite different the Bose and Fermi gases, and some "equivalences" between them could be introduced in the presence of certain conditions, including temperature limitation. This equivalence is usually taken in high temperature limit only. It has been known that at low temperature, ideal gases have a peculiar dependence on the number of dimension $d$. For this reason, the two dimensional (2D) gases may well possess simpler properties than in the one dimensional (1D) gases [1]. The 2D ideal Bose and Fermi gases have the same specific heat at the same temperature [2]. Then, it has been shown that there exists a complete equivalence between the two gases in 2DciteLee. A little bit later, the role played by the spatial dimensionality $d$ is also elucidated and it turns out that there is nothing fundamentally special about dimensionality 2 ; if the single-particle energy spectrum operating in the systems is of the form $\varepsilon \propto p^{s}$, then the equivalence in question arises when and only when $d=s$ [3].

The thermodynamic "equivalence" between noninteracting Bose and spinless Fermi gases in two dimensions, and between one dimensional Bose and Fermi systems with linear dispersion, both in the grand-canonical ensemble is only special cases of a larger class of equivalences of noninteracting systems having an energy-independent single-particle density of states (DOS). The equivalence for systems with a constant DOS is a special case of a more general equivalence 
between noninteracting Bose and Fermi gases with a discrete ladder-type spectrum in the grandcanonical ensemble, which reduces to the constant - DOS case when the level-spacing approaches zero [4].

As we know, metallic carbon nanotubes (CNT) is a quasi-one-dimensional system with zero band gap and respects linear dispersion law. In this paper, we investigate the equivalence between Bose and Fermi gases in this case.

\section{DENSITY OF STATES DOS OF METALLIC CARBON NANOTUBES}

In CNT-like in graphene- the valence and the conduction band cross a the Fermi level.

In the k.p approximation [5] the energy bands of the (n, n) metallic carbon nanotubes are

$$
\varepsilon^{c, v}(n, k)= \pm \gamma \sqrt{\Delta(n)^{2}+k^{2}},
$$

where $\Delta(n)=\Delta n, \Delta=2 \pi / L$ is the half energy gap, $L$ is circumference of the CNT, $\gamma=\sqrt{3} a \gamma_{0} / 2=$ $\hbar v_{F}, v_{F}$ is the Fermi velocity.

For $n=0: \varepsilon^{c, v}(0, k)= \pm \gamma k$, and for $n=1: \varepsilon^{c, v}(1, k)= \pm \gamma \sqrt{\Delta^{2}+k^{2}}$.

The gap between conduction (c) and valence (v) bands $\varepsilon_{\text {gap }}=2 \Delta=4 \pi / L$.

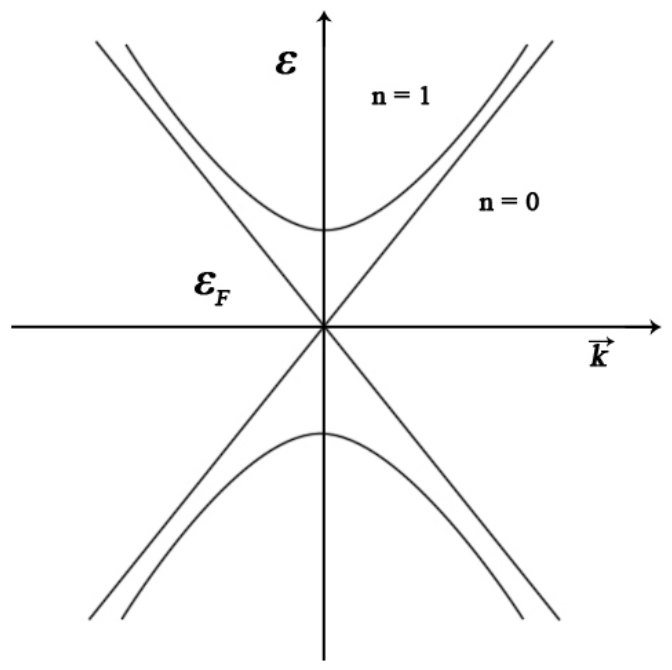

Fig. 1. Energy band structure of metallic carbon nanotubes.

Thus, density of states (DOS) of metallic carbon nanotubes is

$$
g(n)=\frac{1}{L} \frac{\partial N}{\partial \varepsilon}=\frac{1}{\pi \gamma^{2}} \frac{1}{\sqrt{\frac{1}{\gamma^{2}}-\frac{\Delta(n)^{2}}{\varepsilon^{2}}}}
$$

for $\mathrm{n}=0,-\Delta<\varepsilon<\Delta: \mathrm{g}(0)=\frac{1}{\pi \gamma}$,

for $\mathrm{n}=1,-2 \Delta<\varepsilon<-\Delta$ or $2 \Delta>\varepsilon>\Delta: \mathrm{g}(1)=\frac{1}{\pi \gamma^{2}} \frac{1}{\sqrt{\frac{1}{\gamma^{2}}-\frac{\Delta}{\varepsilon^{2}}}}$. 
When $n=0$, DOS $g(0)$ is a constant value, independent on energy $g(0)=C s t$, with $C s t=\frac{1}{\pi \gamma}$.

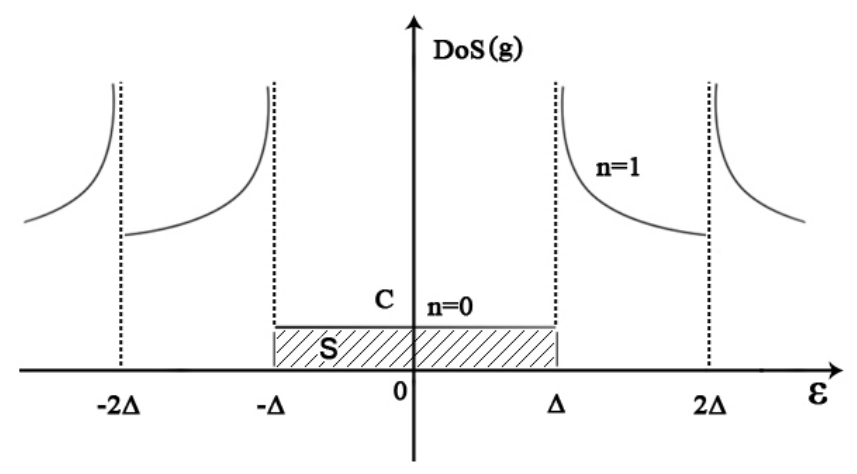

Fig. 2. Density of States (DOS) of metallic carbon nanotubes for $n=0$ and $n=1$ levels.

\section{THERMODYNAMIC EQUIVALENT OF NON-INTERACTING BOSE AND FERMI GASES}

Under low doping regime, particle concentration (electron or hole) is low, the absolute value of Fermi energy is small in comparing with the gap $\left|\varepsilon_{F}\right|<<\Delta$, the Fermi gas can be considered as non-interacting.

The free energy $F_{F}$ and $F_{B}$ of the Fermi and Bose systems, respectively, are related by

$$
F_{F}(T, V, N)-F_{B}(T, V, N)=\frac{N^{2}}{2 C}
$$

where $C$ is a constant that may depend on the system volume $V$, but is independent of the temperature $T$ and the mean particle number $N$.

Consider the gas systems of Bose particle and Fermi particle in a metallic carbon nanotube. Assume that the two system has the same volume $V$, temperature $T$ and the mean particle number $N$ with a constant DOS Thermodynamic.

The entropies of the Fermi and Bose systems are identical [4]

$$
S_{F}(T, V, N)=S_{B}(T, V, N)
$$

The chemical potentials are simply shifted by a temperature-independent constant.

The grand-canonical partition function of an arbitrary noninteracting Bose or Fermi system could be written as [4]

$$
Z=\prod_{\alpha} \sum_{N_{\alpha}} e^{-\beta\left(\varepsilon_{\alpha}-\mu\right) N_{\alpha}}
$$

with $\alpha$ is the labels the quantum states of a single the Bose or the spinless Fermi particle with spectrums $\varepsilon_{\alpha}$, and $\beta \equiv \frac{1}{k_{B} T}, N_{\alpha}=0,1,2 \ldots$ for the bosons and $N_{\alpha}=0,1$ for the fermions. 
The thermodynamic potential $\Omega \equiv F-\mu N$ is given by [4]

$$
\Omega=-\frac{1}{\beta} \ln Z \text {. }
$$

The average number of particles is required to be the same for the Bose and Fermi cases, their chemical potentials $\mu$ in Eq (7) are different. The relations between $\mu_{B}, \mu_{F}$ and $\mathrm{N}$ are determined by

$$
\sum_{\alpha} n_{B}\left(\varepsilon_{\alpha}-\mu_{B}\right)=\sum_{\alpha} n_{F}\left(\varepsilon_{\alpha}-\mu_{F}\right)=N
$$

where $n_{B}, n_{F}$ are the Bose and Fermi distribution functions.

For two systems with a constant DOS, the equivalence of entropies could be directly demonstrated. The Bose and Fermi entropies are [6]

$$
S_{B}=-C k_{B} \int_{0}^{\infty} d \varepsilon\left[n_{B}\left(\varepsilon-\mu_{B}\right) \ln \left[n_{B}\left(\varepsilon-\mu_{B}\right)\right]-\left[1+n_{B}\left(\varepsilon-\mu_{B}\right)\right] \ln \left[1+n_{B}\left(\varepsilon-\mu_{B}\right)\right]\right]
$$

and

$$
S_{F}=-C k_{B} \int_{0}^{\infty} d \varepsilon\left[n_{F}\left(\varepsilon-\mu_{F}\right) \ln \left[n_{F}\left(\varepsilon-\mu_{F}\right)\right]+\left[1-n_{F}\left(\varepsilon-\mu_{F}\right)\right] \ln \left[1-n_{F}\left(\varepsilon-\mu_{F}\right)\right]\right] .
$$

Changing the integration variable in the Bose case to $\omega=e^{\beta\left(\varepsilon-\mu_{B}\right)}-1$ and in the Fermi case to $\omega=e^{\beta\left(\varepsilon-\mu_{F}\right)}$, we have

$$
S_{B}=C k_{B}^{2} T \int_{z_{B}^{-1}-1}^{\infty} d \omega\left[\frac{\ln (1+\omega)}{\omega}-\frac{\ln \omega}{1+\omega}\right]
$$

and

$$
S_{F}=C k_{B}^{2} T \int_{z_{F}^{-1}}^{\infty} d \omega\left[\frac{\ln (1+\omega)}{\omega}-\frac{\ln \omega}{1+\omega}\right] .
$$

As the average particle numbers are the same, these lower limits coincide and thus the Fermi and Bose entropies are identical.

We also introduce $\eta$ as a degree of equivalence. We investigate the variance of $\eta$ in relation with circumference $L$ of the CNT.

$$
\eta=\frac{S(L)}{S\left(L_{\min }\right)}=\frac{g_{0} .2 \Delta(L(n))}{g_{0} .2 \Delta\left(L_{\min }\right)}
$$

as $\Delta=2 \pi / L(n)$, so $\eta \propto L_{\min } / L(n)$. For metallic carbon nanotube, when $n=3, L_{\min }=L(3)$. The degree of equivalence can be treat as below (Fig. 3)

It's easy to see that $\eta$ attain its $\max =1$ when $L(n)=L_{\min }=L(3) . \eta$ tends to 0 rapidly when $L$ increases.

So, for the carbon nanotube $(3,3)$, the Bose and Fermi gases systems could have the maximum equivalence. 


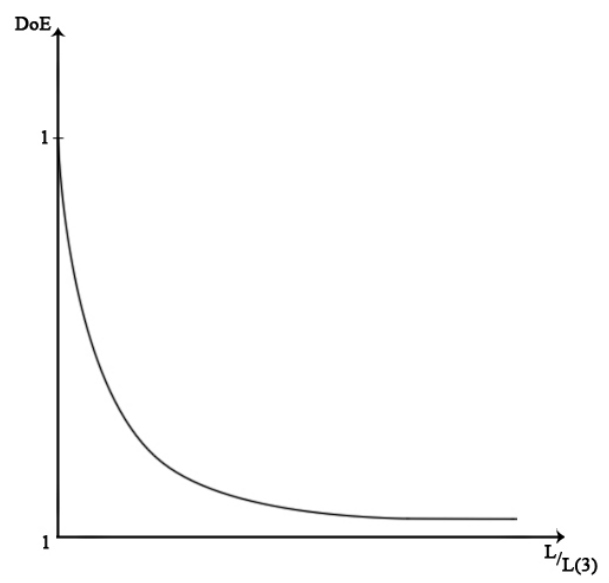

Fig. 3. Dergee of equivalence of metallic carbon nanotubes.

\section{CONCLUSION}

We had presented one more interesting example for the equivalence between Bose and Fermi gases systems. We know that the carbon nanotube could be considered as quasi onedimensional system or not depending on its circumference. When increasing the carbon nanotube circumference, the CNT must be treated in two-dimensional, and has no more the properties of quasi one-dimensional system. In investigating the degree of equivalence, we showed that the validity of equivalency decreases rapidly with the increasing of CNT circumference. The equivalence exists only in the limit of $k$ in the quasi one-dimensional system of metallic carbon nanotube.

It should be also noted that the equivalence could be established for the gases in metallic carbon nanotube only, not for the semi-conductor or kekule structure [7].

At higher doping level, high particle concentration leads to the case of interacting gases. In the Fermi scheme at very high concentration, a Fermi gas in metallic carbon nanotubes may condensate to the Luttinger liquid. The question is that in the according Bose scheme the gas could be faced with Bose-Einstein condensation? And what is link between two types of condensations. We hope to get the answer in next publications.

\section{ACKNOWLEDGMENT}

Part of this work has been supported by the Nafosted Grant No. 103.06-2011.51.

\section{REFERENCES}

[1] M. H. Lee, Phys. Rev. E 55 (1997) 1518.

[2] Robert M. May, Phys. Rev. 135 (1964) A1515-A1518.

[3] R. K. Pathria, Phys. Rev. E 57 (1998) 2697.

[4] Kelly R. Patton, Michael R. Geller and Miles P. Blencowe Physica A 357 (2005) 427-435.

[5] H. Ajiki and T. Ando, J. Phys. Soc. Japan 65, 505 (1996); Physica B 216 (1996) 358.

[6] L. D. Landau and E. M. Lifshits, Statistical Physics, $3^{\text {rd }}$ ( Pergamon Press, Oxford, 1980), Part 1.

[7] A. Viet, H. Ajiki, and T. Ando, J. Phys. Soc. Japan 63 (1994) 3036. 